\title{
Electron Microscopy and Tomography on Endocytosis of Macrophages
}

\author{
I. Ratnayake ${ }^{1}$, S. P. Ahrenkiel ${ }^{1}$, A. Hoppe ${ }^{2}$ and N. Thiex ${ }^{3}$ \\ 1. Nanoscience and Nanoengineering Ph.D. Program, South Dakota School of Mines and Technology, \\ Rapid City, SD, USA \\ 2. Department of Chemistry and Biochemistry, South Dakota State University, Brookings, SD, USA \\ 3. Department of Biology and Microbiology, South Dakota State University, Brookings, SD, USA
}

Macrophages are a type of white blood cell that engulf and digest foreign substances, microbes, and cancer cells in a process called endocytosis, so macrophages act like scavengers. They are constantly roaming around, searching for and destroying dead cells and foreign particles that do not belong in the body. There are three types of endocytosis: phagocytosis, pinocytosis, and receptor-mediated endocytosis. Transmission electron microscopy (TEM) is capable of much higher magnification and greater resolving power than optical microscopy, allowing visualization of much smaller objects with finer detail. Traditional TEM techniques provide only 2D projection views of thin cellular slices. However, electron tomography (ET) technique provides a way for obtaining detailed 3D structures of sub-cellular macromolecular objects [1]. TEM tomography of macrophages is being developed for visualization of the endocytosis process in relation to cell structure.

Differences in the structural characteristics of cells undergoing phagocytosis and pinocytosis were observed by TEM. Figure 1 shows a few important components of a phagocytic macrophage cell. Figure 2 shows a comparison between the structural characteristics of phagocytosis and pinocytosis processes. The phagocytic vesicles are called "phagosomes" and are filled with engulfed solid materials where as the pinocytotic vesicles are called "macropinosomes" and are filled with fluid materials, which have been taken into the cell. Cultured bone-marrow macrophages were prefixed with glutaraldehyde in a stabilization buffer. The cells were post fixed and stained with osmium tetroxide and uranyl acetate successively. The post-fixed cells were dehydrated in a graded series of ethanol solutions and subsequently embedded in an ultraBed low viscosity spur media. Thin sections were cut on an PowerTome XL ultramicrotome and electron microscopy was performed with a JEM-2100 TEM. In particular, we will present a quantitative comparison of vesicle characteristics for three different types of macrophage samples: (1) normal phagocytosis, (2) normal pinocytosis and (3) stimulated pinocytosis. Stimulation increases the cell activity and increases the number of endosomes within each cell as shown in figure 3 . The quantitative analysis between stimulated and non-stimulated cells shows increased activity of the endocytosis process due to treatment.

We also will discuss the clathrin-mediated endocytosis process in macrophages to elucidate the role of clathrin (protein) molecules in maturation and degradation of endosomes in macrophages. We compare, control samples (with clathrin) to knockout cells (without clathrin). For cells in which clathrin has been knocked out, intraluminal vesicles (ILVs) and late endosomes (LE) are present, showing that ILV degradation correlates to the presence of clathrin. Figure 4 shows the TEM images of controlled and knockout cells. 


\section{References:}

[1] Subramaniam, S. \& Zhang, P., ASM News-American 69 (2003), p. 240.

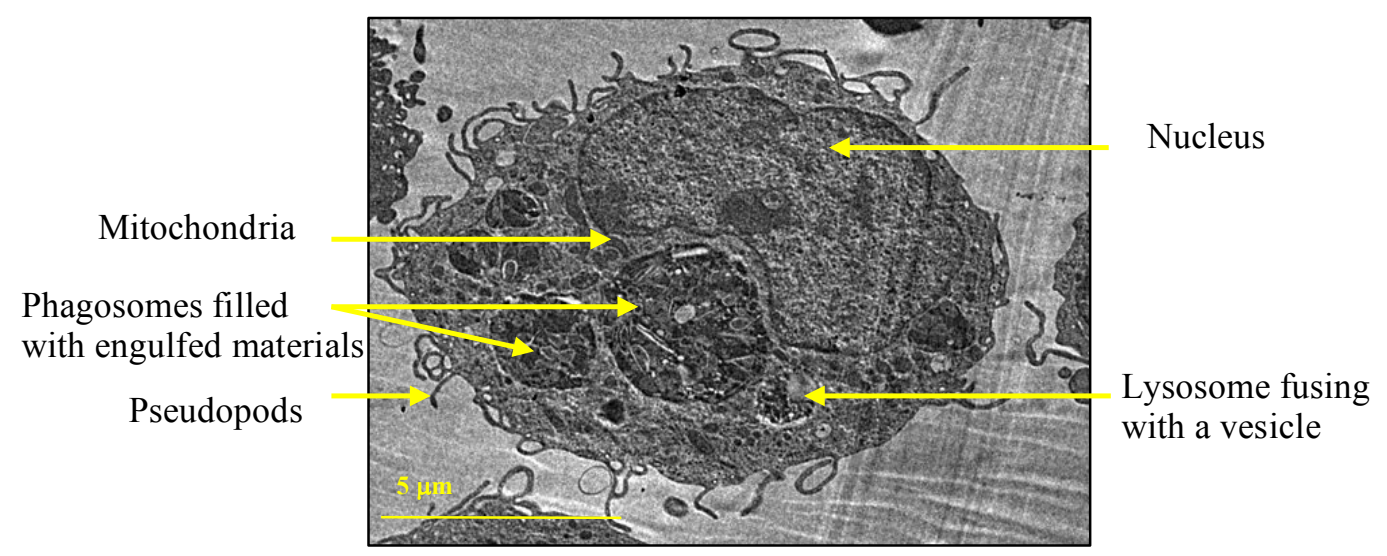

Figure 1. The observed features of a phagocytic macrophage.
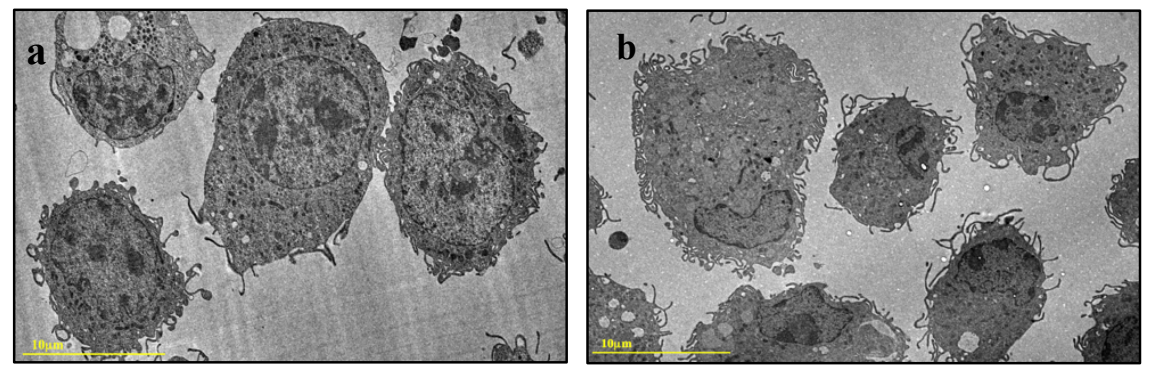

Figure 2. TEM images of: (a) Phagocytic macrophages, and (b) Pinocytotic macrophages.
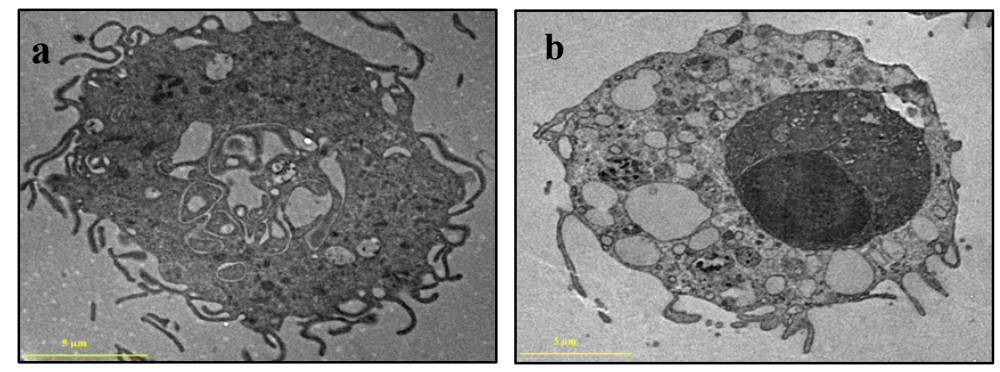

Figure 3. TEM images of pinocytotic macrophages: (a) Non-stimulated cell, and (b) Stimulated cell.
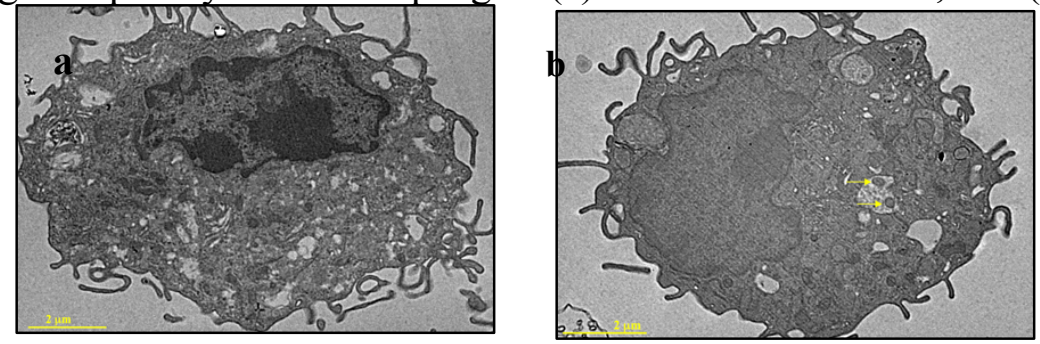

Figure 4. TEM images of a: (a) Controlled cell, and (b) Knockout cell with the presence of ILVs inside LEs (arrows) 